\title{
On the role of virtual work in Levi-Civita's parallel transport
}

\author{
Giuseppe Iurato, University of Palermo, Italy; e-mail: giuseppe.iurato@unipa.it
}

Giuseppe Ruta, Sapienza University, Rome, Italy; e-mail: giuseppe.ruta@uniroma1.it

\begin{abstract}
The current literature on history of science reports that Levi-Civita's parallel transport was motivated by his attempt to provide the covariant derivative of absolute differential calculus with a geometrical interpretation. ${ }^{1}$ Levi-Civita's memoir on the subject was explicitly aimed at simplifying the geometrical computation of the curvature of a Riemannian manifold. In the present paper we wish to point out the possible role implicitly played by the principle of virtual work in Levi-Civita's conceptual reasoning to formulate parallel transport.
\end{abstract}

\section{Introduction}

Tullio Levi-Civita (1873-1941) was one of the leading Italian mathematicians of his time. He showed his attitude towards mathematics and mathematical physics since his high-school days, when he offered an ingenious attempt to prove Euclid's fifth postulate on parallel lines under the supervision of his teacher in mathematics, Paolo Gazzaniga (1853-1930), a scholar in number theory. ${ }^{2}$ Levi-Civita took a degree in mathematics from the university of Padua, where among his teachers he had Giuseppe Veronese (1853-1917), ${ }^{3}$ Francesco Flores d'Arcais (18491927), ${ }^{4}$ Gregorio Ricci-Curbastro (1853-1925), ${ }^{5}$ and Ernesto Padova (1845-1896). ${ }^{6}$ Both RicciCurbastro and Padova provided Levi-Civita with a strong training in mathematical physics and mechanics. Ricci-Curbastro, in particular, provided him with firsthand absolute differential calculus methods, which were the inheritance of Beltrami's investigations on Riemannian manifolds [16, 38]. Levi-Civita applied these methods to subjects other than differential geometry, such as mechanics. In 1902, he took over the chair of higher mechanics in Padua, vacant after Padova's premature death. In the some twenty years he spent in Padua until he was appointed to Rome, Levi-Civita investigated a vast set of subjects in analytical mechanics. In the mid-1910s Levi-Civita turned his attention to problems in both special and general relativity, treated analytically with the new powerful tools of the absolute differential calculus to which he had contributed. ${ }^{7}$ In particular, he studied the curvature of four-dimensional Riemannian manifolds, modelling space-time through the parallel transport of vectors over these manifolds.

\footnotetext{
${ }^{1}$ For instance, see [36], sect. 4.

${ }^{2}$ Biographical notes are taken from Levi-Civita's obituary by Ugo Amaldi (1875-1957), [29], vol. 1, IX-XXX.

${ }^{3}$ Veronese spent a period of time studying in Leipzig under the supervision of Felix Klein (1849-1825). He provided contributions to projective hyper-spaces and non-Euclidean geometry.

${ }^{4}$ Flores d'Arcais graduated in Pisa, and had Enrico Betti (1823-1892) and Ulisse Dini (1845-1918) among his teachers. He is well known in the Italian school of mathematics of the days for his excellent handbooks on calculus.

${ }^{5}$ Ricci-Curbastro graduated in Pisa having Betti, Dini, and Eugenio Beltrami (1835-1900) among his teachers. He perfected his studies with Klein and, jointly with Levi-Civita, is considered the father of absolute differential calculus.

${ }^{6}$ A pupil of Beltrami, Padova investigated mathematical physics in non-Euclidean spaces.

${ }^{7}$ It is well known that Albert Einstein (1879-1955) claimed to feel indebted with him for absolute calculus.
} 
He took into account the curvature of the manifold by varying some parameters along paths lying on the manifold. Indeed, at every point of the manifold it is possible to consider a linear structure, the tangent space, which can be linked to the tangent spaces at other points of the manifold through a well-determined new mathematical notion, later called a connection, which, thus, originates from parallel transport. ${ }^{8}$

In the mathematical literature we usually read that Levi-Civita's parallel transport was motivated by his attempt to give a geometrical interpretation to the so-called covariant derivative of absolute differential calculus. In addition, according to Nastasi and Tazzioli [33], Levi-Civita defined parallelism on a Riemannian manifold through a new formulation of the law of inertia for a point moving on a geodetic line. ${ }^{9}$ With respect to Levi-Civita's original procedure in [28], we believe that such an interpretation is possible only a posteriori, within the framework of a global variational calculus. The same can be said for the consideration of Levi-Civita's parallelism as a local geometrical interpretation of covariant derivative, as in [34]. ${ }^{10}$ On the other hand, in this paper we point out a possible a priori interpretation of the intrinsic origin of Levi-Civita's parallelism based on the tacit use of schemes of analytical mechanics and the principle of virtual work.

If we read Levi-Civita's paper [28] carefully, we see that Levi-Civita aimed at simplifying the computation of the curvature of a Riemannian manifold by re-examining the covariant behaviour of the Riemann symbols. ${ }^{11}$ In pursuing this, he devoted the first fourteen sections of his memoir to introducing and explaining parallelism on an arbitrary Riemannian manifold $V_{n}$ of dimension $n \geq 2$. From examining these passages, we believe that Levi-Civita's strong education in mathematical physics had a remarkable role in his developments by tacitly influencing his fashion of formal reasoning. This was also due to the fact that, at that time, geometry and mechanics had evanescent boundaries, and a language with many common traits and analogies of meaning.

To begin with, when Levi-Civita considered tangent spaces at the various points of the manifold, it is apparent that he tacitly adopted equations formally analogous to those of virtual displacements compatible with a set of constraints. The implicit mechanical interpretation of a Riemannian manifold as the space of the configurations of a mechanical system with a finite set of degrees of freedom, subjected to a set of holonomic constraints, is indeed possible and conceivable. Even if nowadays this interpretation is standard, see Arnold [4] for instance, we cannot take it for granted at the beginning of the 20th century. Levi-Civita himself remarked that some of his conditions were those of admissible first-order constrained displacements. Nevertheless, this is only one of the possible interpretations of how Levi-Civita could have proceeded in setting up and solving his initial aim of simplifying the computation of Riemann's symbols.

Indeed, Levi-Civita's condition of parallelism is expressed by the vanishing of a linear form built on the tangent space at a point of the manifold: it is apparent that such a statement has many a formal resemblance with the well-known principle of virtual work, deeply rooted in the Italian school of mathematical physics since the pioneering works by Joseph Louis Lagrange (1736-1813). Indeed, the use of various forms of the principle of virtual work may be found in all scholars in Italian mathematical physics and engineering, such as Gabrio Piola (1794-1850),

\footnotetext{
${ }^{8}$ Parallel transport and linear (affine) connection were introduced almost simultaneously by Levi-Civita, Gerhard Hessenberg (1874-1925), Hermann Weyl (1885-1955) and Jan Arnoldus Schouten (1883-1971) in connection with Einstein's general theory of relativity. Levi-Civita used (local) embedding of a Riemannian manifold in some $n$-dimensional space. Weyl introduced parallel transport (hence, linear connection) on arbitrary differential manifolds on a completely general basis, that is, with no reference to Riemann metrics, see [32], ch. 1, sect.
} 1.1 .

${ }^{9}$ Sect. 1.3 , p. 214 .

${ }^{10}$ Sect. 3, p. 337 .

${ }^{11}[28]$, Introduction. 
Betti, Beltrami, Luigi Federico Menabrea (1809-1906), Carlo Alberto Castigliano (1847-1884), Valentino Cerruti (1850-1909) [12, 13, 14, 15, 35, 6, 40, 39]. We cannot but infer that such a key idea had a strong influence on the writings of Levi-Civita, educated at the same school, and this mechanical interpretation of Levi-Civita's condition of parallelism is a possible, plausible historical interpretation. Moreover, it was Levi-Civita himself who provided an additional expression of his differential condition of parallelism in a form that it is exactly like Lagrange's general equation of analytical mechanics. Piola's influence on the school that led to Levi-Civita is put forth and suggested also in [3].

Thus, from our historical analysis it emerges that the principle of virtual work could have been among one of the conceptual guides, or mental schemes, to build up Levi-Civita's notion of parallel transport. This is corroborated also by what we find in Bottazzini [10], where the primary role played by the new results of general relativity is stressed among the motivations of Levi-Civita's memoir of 1917 [28]. In the following, we first sketch some hints of the origin and the various formulation of the principle of virtual work in the history of mechanics. Then we point out its possible conceptual role in the early origin of the formal notion of parallel transport due to Levi-Civita, by examining its presentation in the original paper [28].

\section{Some hints on the principle of virtual work}

Broadly speaking, in modern textbooks of mechanics we find that a system of bodies is balanced if and only if the total power on any admissible (virtual) velocity field vanishes. Passing from power to work means only a rescaling by a small time interval $\delta t$, and we get the principle of virtual work. Since the second half of 18th century, the law of virtual work saw no appreciable changes from the formulation by Lagrange, who in the first edition (1788) of his Mécanique analytique put Johann Bernoulli's principle of virtual velocities at the base of mechanics.

To be precise, in 1788 [23] Lagrange identified three main paradigms in the history of statics, i.e. the lever, the composition of forces, and the principle of virtual work. The paradigm of the lever would have been in force from antiquity until up the early 18th-century, when Pierre Varignon (1654-1722) worked out the (de-)composition of forces [41] by the graphical constructions known as polygon of forces and funicular polygon [15]. The principle of virtual work would become dominant after Lagrange. However, Lagrange's prediction was never fully realized because the principle of virtual work did not replace the (de-)composition of forces, but at most outflanked it [11]. Furthermore, Lagrange's picture is too schematic: indeed, some form of virtual work laws have always existed in mechanics, even though with limited applications. Arabic and Latin mechanics of the Middle Ages called attention to such a law, while in the Renaissance we begin to see different wordings of it, often proposed as unique principle of statics. The process reached its apex with Johann Bernoulli and Lagrange, and the French school adopted Lagrange's principle of virtual work, and applied it to continuum mechanics.

A major difficulty in the formulations of virtual work laws is the status attributed to constraints and their reactions. Before the 18th century, constraints had been treated only as passive elements; after accepting models of matter based on particles considered as centres of forces, constraints began to be thought of as capable of providing forces. ${ }^{12}$ Lagrange stated that "the use of these forces released [us] from [the need of] taking into account the constraints among bodies, and allows one to make use of the laws of motion of free bodies". ${ }^{13}$ In statics, constraint reactions are less problematic, since they can be considered as the forces necessary to maintain the constraint. The first to introduce them in calculations was probably Varignon

\footnotetext{
${ }^{12}$ According to Lagrange [23], in the period 1736-1742 the Bernoullis, Alexis Clairaut (1713-1765) and Leonhard Euler (1707-1783) were among the first to assimilate constraint reactions to active forces.

${ }^{13}$ In [23], 1st ed., p. 179; our translation. See also Capecchi [11], p. 15.
} 
[41]. It was the difficulty of incorporating reactive forces in a consistent mechanical theory that led Johann Bernoulli to formulate a law of virtual work, known after Lagrange as the principle of virtual velocities, which provides a balance criterion without the intervention of these undesirable entities. Johann Bernoulli's statement is "the sum of the powers each of them multiplied by the distance traveled from the point where they are applied, in the direction of this power, will always be zero". ${ }^{14}$ Lagrange in his Mécanique analytique suitably reformulated Bernoulli's principle, shifting from his previous use of the principle of least action. Even today, the principle of virtual work is considered more general than that of least action, because it also allows non-conservative forces to be taken into account. However, as a result of the heated discussions that followed the publication of [23], Lagrange changed his mind, and in the second edition (1811) of the Mécanique analytique he remarked that the principle of virtual work is not obvious enough in itself to be selected as a founding principle. ${ }^{15}$ There were also technical difficulties such as the question of the admissible displacements to consider, and whether they should be compatible with the constraints.

In order to formulate Lagrange's expression of the principle of virtual work, following him we must accept that inertia is another force, and add it to the active ones. Thus, the total force on a particle vanishes, and all problems of dynamics are reduced to those of statics. ${ }^{16}$

In modern language, Lagrange's principle of virtual work states that a system of particles is balanced when the active forces ("puissances", i.e., powers) $\vec{F}_{i}$ to which it is subjected satisfy ${ }^{17}$

$$
\delta L=\sum_{i} \vec{F}_{i} \cdot \delta \vec{P}_{i}=0
$$

where $\delta \vec{P}_{i}$ is the first-order displacement of the point of application of $\vec{F}_{i}$. Thus, if a system is in equilibrium, the virtual work of all active forces $\vec{F}_{i}$ will vanish for any virtual displacement.

To keep into account constraint reactions $\vec{R}_{j}$ also, we must add their work to that of the active forces in eq. (1). With this aim, we add the definition, implicitly assumed by Lagrange, of smooth constraints. In modern terms, we assume that the work of the reactions due to smooth constraints is non-negative for any irreversible virtual displacement, while is zero for any reversible virtual displacement. ${ }^{18}$ In the special, yet fundamental, case of bilateral constraints, expressed by equalities, all compatible virtual displacements are reversible. Hence, we assume that the virtual work of constraint reactions is zero, then the principle of virtual work reads

$$
\delta L=0
$$

also called symbolic equation of statics. ${ }^{19}$ In eq. (2), the virtual work is spent by active forces plus inertia, since smooth constraints spend no work on admissible displacements.

If the constraints are holonomic, they are expressed as equalities in the intrinsic parameters of the system, and the vanishing of the virtual work of constraint reactions assumes an interesting expression. This will become apparent in the following, where we consider how such an expression may have probably influenced Levi-Civita's notion of parallel transport.

\footnotetext{
${ }^{14}[23], 1$ st ed., p. 11; our translation.

15 [23], 3rd ed., p. 21.

${ }^{16}$ Lagrange claims such a thesis is D'Alembert's, but D'Alembert's actual principle is different [11].

${ }^{17}[23]$, 1st ed., p. 14.

${ }^{18}$ See [26], vol. 1, ch. XV; vol. 2, part 1, ch. V, sect. 3, n. 18-21; [27], part 1, ch. XIV, sect. 2, n. 4-8; part 2, ch. V, sect. 3 , n. 17-19; [1], vol. 2, ch. V, sect. 1, n. 4; [2], ch. I, sects. 1-2; [20], vol. 1, ch. XIII, sect. 4.

${ }^{19}$ Also said to be D'Alembert-Lagrange principle as reformulated by Lagrange, [4], ch. IV, or general equation of virtual work, [5], vol. I, ch. XV, sect. 318. See also the references in the previous footnote.
} 


\section{Levi-Civita's parallel transport}

In [28], Levi-Civita introduced his contribution by claiming that the absolute differential calculus opened the way to Einstein's theory of gravitation; and, since handling such technicalities was so important, he found it necessary to simplify the calculation of intrinsic curvature. Thus, he went on, it was fundamental to begin with the notion of parallelism in a Riemannian manifold. ${ }^{20}$

Levi-Civita started by considering two directions $\vec{\alpha}, \vec{\alpha}^{\prime}$ emerging from two infinitesimally near points $P, P^{\prime}$ of the Riemannian manifold $V_{n}$, embedded in an $N$-dimensional Euclidean space $S_{N}$.

In $S_{N}$, the two directions $\vec{\alpha}, \vec{\alpha}^{\prime}$ are parallel if

$$
\operatorname{angle}(\widehat{\vec{\alpha}, \vec{f}})=\operatorname{angle}\left(\widehat{\vec{\alpha}^{\prime}, \vec{f}}\right)
$$

for any auxiliary direction $\vec{f}$ emerging from $P$. Parallelism on $V_{n}$ is defined by requiring that the condition in eq. (3) holds for any direction $\vec{f}$ of the plane $T_{P}^{S_{N}}\left(V_{n}\right)$ tangent to $V_{n}$ at $P$. This definition is intrinsic, since it depends on metrics in $V_{n}$, not in the embedding space $S_{N}$.

Levi-Civita emphasized that this condition depends on the path joining $P$ with $P^{\prime}$, being independent only in Euclidean spaces. Along a geodetic line, the tangents keep their direction: this is a generalization of an intuitive property of a straight line in a Euclidean space. ${ }^{21}$

The key idea underlying parallelism in a Riemannian manifold, according to Levi-Civita, is therefore expressed by eq. (3) for two arbitrary concurrent unit vectors (germs of directions in modern language), the origin of which moves along an arbitrary path on the manifold.

Levi-Civita considered generic metrics ${ }^{22}$ on an arbitrary finite-dimensional manifold ${ }^{23} V_{n}$

$$
d s^{2}=\sum_{i, k=1}^{n} a_{i k} d x_{i} d x_{k}
$$

and embedded $V_{n}$ in a Euclidean space $S_{N}$ with sufficiently great dimension $N \leq n(n+1) / 2$, so that it may be described by the system ${ }^{24}$

$$
y_{\nu}=y_{\nu}\left(x_{1}, \ldots, x_{n}\right), \quad \nu=1,2, \ldots, N
$$

where the $y_{\nu}$ are coordinates in $S_{N}$, while the $x_{n}$ are intrinsic coordinates on $V_{n}$. Remark that eq. (5) describes also a discrete mechanical system with $n$ degrees of freedom subjected to $N$ smooth holonomic bilateral constraints. This is a key point of our possible interpretation of Levi-Civita's parallelism notion: the shift from a point on $V_{n}$ to one nearby is subjected to eq. (5), hence its expression is analogous to the admissible velocity field (or, modulo a rescaling, to the field of virtual displacements) of an analog constrained mechanical system. It seems to us that Levi-Civita's education in mathematical physics clearly emerges here.

Levi-Civita considered an arbitrary direction of $S_{N}$ by the unit vector $\vec{f}$, with direction cosines $f_{\nu}$, and another arbitrary direction at a point $P$ of $V_{n}$. This last is characterized by the unit vector $\vec{\alpha}$ with direction cosines $\alpha_{\nu}$ with respect to $S_{N}$ (of course, since $V_{n}$ is embedded in the ambient space $S_{N}$, each direction belonging to $V_{n}$ also belongs to $S_{N}$.).

\footnotetext{
${ }^{20}$ See also [34], sect. 3 .

${ }^{21}[28]$, p. 3.

${ }^{22}$ In doing so, Levi-Civita dealt with first forms of pseudo-Riemannian structures, see Bottazzini [10], pp. 305-306.

${ }^{23}$ [9], ch. XXV.

${ }^{24}[28]$, eq. (1), p. 4.
} 
The point $P$ may be thought of as varying on a smooth curve $\mathcal{C}$ lying on $V_{n}$, parameterized by the abscissa $s$ in eq. (4), thus $\alpha_{\nu}=\alpha_{\nu}(s)$. Let $x_{i}=x_{i}(s), i=1,2, \ldots, n$ be the intrinsic parametric equations of $\mathcal{C}$. Then, $\mathcal{C}$ may also be represented by the parametric equations $y_{\nu}=y_{\nu}(s), \nu=1, \ldots, N$ when it is thought of as embedded in $S_{N}$ via eq. (5). Indeed, since $x_{i}=x_{i}(s), i=1, \ldots, n$, it is possible to write

$$
\mathcal{C} \equiv y_{\nu}(s)=y_{\nu}\left(x_{1}(s), \ldots, x_{n}(s)\right), \nu=1, \ldots, N .
$$

It is apparent that, in the analog constrained system, $s$ is an evolution parameter that may be thought to coincide with time, and $\mathcal{C}$ is a trajectory in the manifold of admissible configurations.

To find the unit direction emerging from a point $P$ of $\mathcal{C}$, Levi-Civita derived its parametric representation, given by eq. (6), with respect to the natural abscissa $s^{25}$

$$
y_{\nu}^{\prime}=\sum_{i=1}^{n} \frac{\partial y_{\nu}}{\partial x_{i}} x_{i}^{\prime} \quad \nu=1,2, \ldots, N,
$$

where a prime indicates a derivative with respect to $s$. Thus, Levi-Civita obtained the direction cosines with respect to $S_{N}$, while $x_{i}^{\prime}$ are the direction cosines of the same unit direction with respect to $V_{n}$.

Levi-Civita supposed that at each point $P$ of $\mathcal{C}$ there is a direction $\vec{\alpha}$ of $V_{n}$, the direction cosines of which are $\xi^{(i)}, i=1,2, \ldots, n$ with respect to $V_{n}$, and $\alpha_{\nu}, \nu=1, \ldots, N$ with respect to $S_{N}$. Then, eq. (7) yields ${ }^{26}$

$$
\alpha_{\nu}=\sum_{l=1}^{n} \frac{\partial y_{\nu}}{\partial x_{l}} \xi^{(l)} \quad \nu=1,2, \ldots, N .
$$

which is a linear form on the direction cosines of the direction $\vec{\alpha}$ of $V_{n}$.

When $P$ varies along $\mathcal{C}$, ordinary parallelism in $S_{N}$ implies the equality of the angle between $\vec{\alpha}$ and a direction $\vec{f}$ arbitrarily chosen. Levi-Civita introduced an intrinsic notion of parallelism in $V_{n}$ by considering two nearby points. He considered an arbitrarily fixed direction $\vec{f}$ of $S_{N}$, the direction cosines of which are $f_{\nu}$, and the cosine of the angle between $\vec{f}$ and $\vec{\alpha}$

$$
\cos (\widehat{\vec{f}, \vec{\alpha}})=\sum_{\nu=1}^{N} \alpha_{\nu} f_{\nu}
$$

Then, he considered an infinitesimal variation $d s$ of the natural abscissa $s$ on $V_{n}$, which implies that the cosine provided by eq. (9) undergoes the variation

$$
d \cos (\widehat{\vec{f}, \vec{\alpha}})=d s \sum_{\nu=1}^{N} \alpha_{\nu}^{\prime}(s) f_{\nu} .
$$

The ordinary parallelism between $\vec{\alpha}$ and $\vec{f}$ would require eq. (10) to vanish when $\vec{f}$ varies in $S_{N}$, implying $\alpha_{\nu}$ to be uniform. Levi-Civita, however, imposed, as an intrinsic condition of parallelism, the weaker condition that the angle between $\vec{\alpha}$ and $\vec{f}$ be constant when $\vec{f}$ varies on $V_{n}$. That is, he supposed the variation in eq. (10) to be zero only for the directions that are tangent to $V_{n}$ as $P$ varies along $\mathcal{C}$.

Levi-Civita claimed ${ }^{27}$ that these directions are exactly those compatible with the constraints (5). According to us, once again this is a hint that he had clearly in mind the mechanical analogy

\footnotetext{
${ }^{25}[28]$, eq. (4), p. 5.

${ }^{26}[28]$, eq. (7), p. 6 .

$27[28]$, p. 7.
} 
with his geometrical investigation. Thus, by replacing the $f_{\nu}$ with quantities proportional to them, Levi-Civita's intrinsic definition of parallelism implies ${ }^{28}$

$$
\sum_{\nu=1}^{N} \alpha_{\nu}^{\prime}(s) \delta y_{\nu}=0
$$

for any variation $\delta y_{\nu}$, that is, in the analog holonomic discrete mechanical system, for any admissible displacement compatible with the constraints in eq. (5). With a suitable mechanical interpretation of the $\alpha_{\nu}^{\prime}(s)$, for instance by considering them as a kind of mechanical action in $S_{N}$, eq. (11) is a formulation of the virtual work principle in $S_{N}$ related to the smooth bilateral holonomic system defined by eq. (5), hence related to a Riemannian manifold. ${ }^{29}$ As already said, in this paper we wish to emphasize this formal analogy, which might conceptually have led Levi-Civita to work out his intrinsic geometrical notion of parallelism.

From eq. (5) it follows that ${ }^{30}$

$$
\delta y_{\nu}=\sum_{k=1}^{n} \frac{\partial y_{\nu}}{\partial x_{k}} \delta x_{k} \quad \nu=1,2, \ldots, N
$$

with $\delta x_{k}$ arbitrary, so that eq. (11) reduces to ${ }^{31}$

$$
\sum_{\nu=1}^{N} \alpha_{\nu}^{\prime}(s) \frac{\partial y_{\nu}}{\partial x_{k}}=0 \quad(k=1,2, \ldots, n),
$$

which are the formal conditions for the parallelism of the directions $\vec{\alpha}$ moving along $\mathcal{C}$. Nevertheless, in eq. (13) there are also parameters regarding $S_{N}$, so that, in order to have an intrinsic definition, it is necessary to involve only parameters regarding $V_{n}$. To this aim, one has to replace the direction cosines $\alpha_{\nu}$ with their expression given by eq. (8), so to involve the intrinsic direction cosines $\xi^{(i)}$, and to deduce ${ }^{32}$

$$
\frac{d \xi^{(i)}}{d s}+\sum_{j, l=1}^{n} \Gamma_{i}^{j l} x_{j}^{\prime} \xi^{(l)}=0 \quad(i=1,2, \ldots, n)
$$

where $\Gamma_{i}^{j l}$ are Christoffel symbols of second kind, defined as follows ${ }^{33}$

$$
\Gamma_{i}^{j l}=\sum_{k=1}^{n} a^{i k}\left(\frac{\partial a_{k l}}{\partial x_{j}}+\frac{\partial a_{j k}}{\partial x_{l}}-\frac{\partial a_{j l}}{\partial x_{k}}\right) \quad(i, j, l=1,2, \ldots, n),
$$

where the $a^{i k}$ are the components of the reciprocal form of (4). Levi-Civita showed that eq. (14) may be expressed by the covariant quantities associated with the $\xi^{(i)}, 34$ so that the intrinsic condition of parallelism has the same form of Lagrange equations of motion on a Riemannian manifold. ${ }^{35}$

\footnotetext{
${ }^{28}[28]$, eq. (I), p. 7.

${ }^{29}[21]$, ch. 3 , sect. 2 , n. 2.6 .

${ }^{30}[28]$, unnumbered equation before eq. eq. (8), p. 7.

31 [28], eq. (8), p. 7.

$32[28]$, eq. $\left(\mathrm{I}_{a}\right)$, p. 8 .

33 [29], vol. IV, p. 8, [8], ch. II.

${ }^{34}$ Levi-Civita used the term 'moment', which was traditional in the Italian school of mathematical physics of his time and denoted a mechanical action dual to a Lagrangian parameter of admissible (virtual) displacements.

${ }^{35}[28]$, eq. $\left(\mathrm{I}_{c}\right)$, p. 12 . The same comment on eq. (14) may be found in later textbooks of the Italian school of mathematical physics, e.g. [19], ch. X.
} 
Thus, the intrinsic conditions characterizing Levi-Civita's notion of parallel transport of the direction $\vec{\alpha}$ along $\mathcal{C}$ as a function of its directional parameters $\xi_{1}, \ldots, \xi_{n}$ with respect to $V_{n}$ lies only within the framework of absolute differential calculus. Furthermore, in the following sections of [28], Levi-Civita did not make any explicit mention to covariant derivatives, except for a hint to Ricci-Curbastro's rotation coefficients. ${ }^{36}$ Then, Levi-Civita applied parallel transport to a Riemannian manifold by making it possible to compute its curvature. So, it seems apparent to us that, at least up to the definition of intrinsic parallelism, one possible key guide to Levi-Civita was mathematical physics, not only pure geometry, and, in particular, the principle of virtual work for a mechanical system subject to smooth holonomic bilateral constraints, which may be considered as a formal guide-scheme in deducing geometrical features.

\section{$4 \quad$ Final remarks}

From a historical standpoint, we claim that the virtual work principle played a possibly remarkable conceptual role in the origin of Levi-Civita's notion of parallel transport in a Riemannian manifold as expressed by eq. (11) and its consequences. Coherently with his mathematicalphysical education, Levi-Civita used a language which clearly referred to this principle and its formal schemata, for example when he used the term 'constraint' and the expression 'displacements compatible with constraint'. Furthermore, he specified that eq. (11) was obtained "for all the displacements $\delta y_{\nu}$ compatible with the constraints (5)" (our translation), and emphasized this sentence in italics (see [28]). This is, according to us, a sign of a clear wish to refer to the so-called symbolic equation of statics for an ideal mechanical system moving along a line $\mathcal{C}$ of a Riemannian manifold. Such an equation is a nullity condition expressing constitutive properties of smooth constraint reactions. ${ }^{37}$ Indeed, for the analog constrained mechanical system, the unit directions emerging from the points of $V-n$ assume the role of admissible displacements, and the dual forms on them are reactions provided by the geometrical links between the elements of the mechanical system. ${ }^{38}$

In a series of Spanish conferences held by Levi-Civita in 1921 the reference to analytical mechanics is more explicit. ${ }^{39}$ Indeed, in discussing parallelism and curvature in a general Riemannian manifold, Levi-Civita stated ${ }^{40}$ that the geometric notion of parallelism resembles the physical notion of work. This is to say that geometric parallelism, in its analytical substratum, is similar to the integral of the differential form $X_{1} d x_{1}+X_{2} d x_{2}$. Starting from this initial physical framework, Levi-Civita provided a purely analytical treatment of his notion of vectorial equipollence upon a surface. Then, he discussed infinitesimal parallelism and its analytical expression by arguing in terms of vector analysis, and reached a simple, characteristic formal condition of parallelism on surfaces, given by the scalar product $\tau \times d \mathbf{u}=0,{ }^{41}$ where $\tau$ is a generic unit vector of the tangent plane to the given surface, and $d \mathbf{u}$ is the first-order infinitesimal variation of a generic vector $\mathbf{u}$ belonging to the same tangent plane.

Afterwards, in [30], sect. IV, p. 103, Levi-Civita started to speak explicitly of "virtual displacements" and "symbolic equation" (we believe that he understood "of statics"), and the virtual displacements were called into question many times in his formal arguments, until he reached an equivalent formal expression of $\tau \times d \mathbf{u}=0$, that is to say, $\delta P \times d \mathbf{u}=0,{ }^{42}$ which

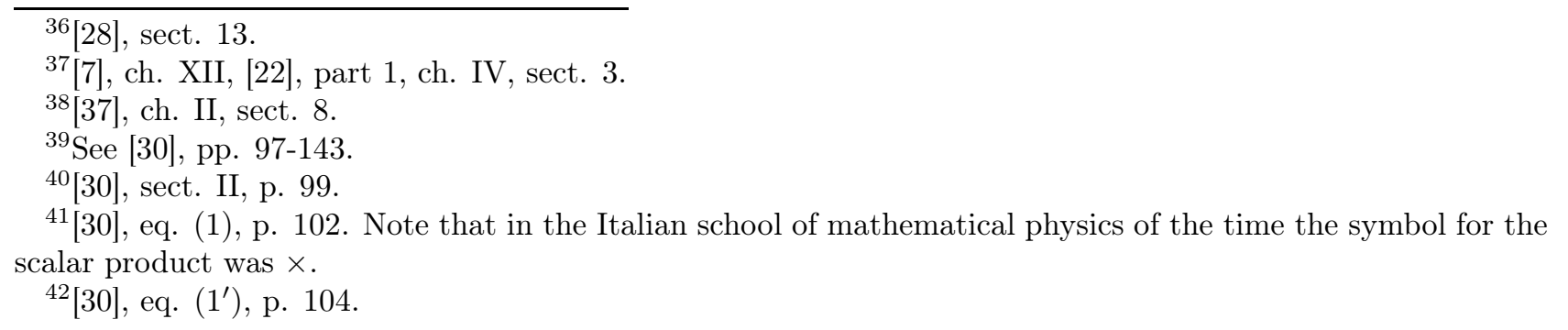


recalls - Levi-Civita stated - the principle of virtual work ([30], sect. IV, p. 104). From this point onwards, Levi-Civita used arguments of analytical mechanics, following a method similar to one that applied the virtual work law to build up Lagrange's equations ([30], sect. IV, p. 109). Thus, he deduced equations (14) for a two-dimensional surface. In [30], sect. VIII, Levi-Civita generalized his results to an arbitrary Riemannian manifold with a simple dimensional extension of the formal model just worked out in a two-dimensional environment, until he had generalized the notion of parallelism to an arbitrary manifold in [30], sect. IX. Then, he deduced equation (11), which now he stated to be "valid for all virtual displacements" ([30], sect. IX, p. 122).

In Levi-Civita's monograph devoted to absolute differential calculus, ${ }^{43}$ we find further $e x-$ post confirmation to our possible historical interpretation. Indeed, in this monograph there are many references to the possible kinematical characterization of parallelism in a Riemannian manifold. Levi-Civita also quoted works from the early 1920s by Giuseppe Corbellinit ${ }^{44}$ and Enrico Persico (1900-1969) ${ }^{45}$ on other possible kinematical interpretations of his own intrinsic parallelism making use of the geometry of developable surfaces. ${ }^{46}$ Levi-Civita further emphasized the possible role of analytical mechanics in developing notions of differential geometry such as that of parallelism, reaching as far as a formal equation characterizing his notion of parallelism, equivalent to (and called by him) the symbolic equation of parallelism. Furthermore, and this is very important for our argument, Levi-Civita himself stated that this equation formally recalls the principle of virtual work. In addition, Levi-Civita used the same principle in deducing another formal property of Riemannian geometry applied to Einstein's general relativity, for instance when discussing the geodetic principle for the dynamics of a material particle moving in a four-dimensional space-time manifold. ${ }^{47}$

Thus, we have shown that the great tradition of the Italian school of mathematical physics of the second half of the 19th century continued in the early decades of the 20th century to produce important results also in the field of 'pure' mathematics that were strongly connected with the most advanced physical issues of the time, i.e., Einstein's theory of general relativity ([10], ch. XVI, sect. 3).

We have stressed what possible formal schemata Levi-Civita followed in deducing his intrinsic, geometric notion of parallelism in an arbitrary Riemannian manifold, starting from the two-dimensional model provided by a material point moving along a smooth surface according to analytical mechanics. Our historical reconstruction of the possible mental route followed by Levi-Civita in working out this primary notion of differential geometry is corroborated by both the formal analysis of the content of his original 1917 memoir (in which our interpretation is supported by the identification of an implicit use of a formalism analogous to that of analytical mechanics) and by the analysis of two main texts of Levi-Civita drawn up soon after: his 1921 contribution to a Spanish conference (included in [30]) and his 1923 lectures recollected in [24].

\section{References}

[1] C. Agostinelli, A. Pignedoli. Meccanica razionale. 2 voll., Zanichelli, Bologna, 1961.

\footnotetext{
${ }^{43}[24]$, ch. V, (b), sects. 10-15. This monograph was suggested by the mathematician Edmund Taylor Whittaker (1873-1956), who wanted to widen the first Italian edition of 1925 with the addition of a third part, devoted to the physical application of absolute differential calculus to Einstein's general relativity, extracted from [25].

${ }^{44}$ We could not find any biographical data on him so far, apart from the fact that he published some notes on intrinsic parallelism.

${ }^{45}$ One of Levi-Civita's pupils, who edited his lectures on the absolute differential calculus, [24].

${ }^{46}[24]$, pp. $102-104$ (pp. 119-121 of the Italian edition).

${ }^{47}[24]$, ch. XI, sect. 12. See [18] for a historical account of the influence of Levi-Civita's parallelism on differential geometry.
} 
[2] C. Agostinelli, A. Pignedoli. Meccanica analitica. 2 voll., Tipografia Mucchi, Modena, 1988.

[3] U. Andreaus, F. dell'Isola, and L. Placidi. At the origins and in the vanguard of peri-dynamics, non-local and higher gradient continuum mechanics. An underestimated and still topical contribution of Gabrio Piola. Mechanics and Mathematics of Solids, 20 (2015), 887-928.

[4] V.I. Arnold. Matematicheskie metody klassicheskoi mekhaniki. MIR, Moscow, 1978. Italian translation: Metodi matematici della meccanica classica. Editori Riuniti, Roma, 1986.

[5] O. Belluzzi. Scienza delle costruzioni. 4 voll., Zanichelli, Bologna, 1961.

[6] E. Benvenuto. An Introduction to the History of Structural Mechanics. Springer Verlag, BerlinNew York, 1991.

[7] G. Bernardini. Fisica generale. 14th Ed., Eredi Virgilio Veschi, Roma, 1974.

[8] L. Bianchi. Geometria differenziale. Volume 1, Parte 1, Zanichelli, Bologna, 1924.

[9] L. Bianchi. Geometria differenziale. Volume 2, Parte 2, Zanichelli, Bologna, 1924.

[10] U. Bottazzini. Il Flauto di Hilbert. Storia della matematica moderna e contemporanea. UTET Libreria, Torino, 1990.

[11] D. Capecchi. History of Virtual Work Laws. Birkhäuser, Boston, 2012.

[12] D. Capecchi, G. Ruta. Piola's contribution to continuum mechanics. Archive for History of Exact Sciences, 61 (2007), 303-342.

[13] D. Capecchi, G. Ruta. A historical perspective of Menabrea's "principle of elasticity". Meccanica, 45 (2010), 199-212.

[14] D. Capecchi, G. Ruta. Cerruti's treatment of linear elastic trusses. Meccanica, 46 (2011), 1283-98.

[15] D. Capecchi, G. Ruta. Strength of Materials and Theory of Elasticity in 19th Century Italy. Springer Int. Publ., 2015.

[16] D. Capecchi, G. Ruta. Beltrami and mathematical physics in non-Euclidean spaces. GAMM 2015 - Proceedings of the 86th annual meeting of the International Association for Mathematics and Mechanics, Lecce (Italy), March 2015.

[17] G. Colonnetti. I fondamenti della statica. Introduzione alla scienza delle costruzioni. UTET, Torino, 1927.

[18] E.T. Davies, K. Yano. The influence of Levi-Civita's notion of parallelism on differential geometry. In: Tullio Levi-Civita. Convegno Internazionale Celebrativo del Centenario della Nascita, Roma, 17-19 Dicembre 1973, Accademia Nazionale dei Lincei, Roma, 1975, pp. 53-76.

[19] B. Finzi and M. Pastori. Calcolo tensoriale e applicazioni. 2nd ed., Zanichelli, Bologna, 1960.

[20] B. Finzi. Meccanica razionale. 2 voll., 3rd ed., Zanichelli, Bologna, 1968

[21] R. Grassini. Meccanica razionale. Liguori, Napoli, 1988.

[22] G. Krall. Meccanica tecnica delle vibrazioni. Zanichelli, Bologna, 1940.

[23] J.L. Lagrange. Méchanique analitique. Desaint, Paris, 1788. 2nd ed.: Mécanique analytique. Courcier, Paris, 1811-1815. 3rd ed.: Mécanique analytique, revue, corrigée, et annotée par M.J. Bertrand. Mallet-Bachelier, Paris, 1853. 
[24] T. Levi-Civita. The Absolute Differential Calculus. Calculus of Tensors. Edited by Enrico Persico, Blackie \& Son, Ltd., London and Glasgow, 1927. Italian edition: Lezioni di calcolo differenziale assoluto. Raccolte e compilate a cura di Enrico Persico, Alberto Stock-Editore, Roma, 1925.

[25] T. Levi-Civita. Fondamenti di Meccanica Relativistica. Redatti da Enrico Persico, Zanichelli, Bologna, 1928.

[26] T. Levi-Civita, U. Amaldi. Lezioni di meccanica razionale. Ristampa anastatica della nuova edizione riveduta e corretta del 1949, Zanichelli, Bologna, 1987-89.

[27] T. Levi-Civita, U. Amaldi. Compendio di meccanica razionale. Ristampa anastatica della seconda edizione riveduta del 1938, Zanichelli, Bologna, 1965.

[28] T. Levi-Civita. Nozione di parallelismo in una varietà qualunque e conseguente specificazione geometrica della curvatura riemanniana. Rendiconti del Circolo Matematico di Palermo, XLII (1917), 173-215. In: Opere IV, 1-39.

[29] T. Levi-Civita. Opere matematiche. Memorie e note. 6 voll., Zanichelli, Bologna, 1954-73.

[30] T. Levi-Civita. Questioni di Meccanica Classica e Relativistica. Zanichelli, Bologna, 1924.

[31] R. Marcolongo. Meccanica razionale. 2 voll., Hoepli, Milano, 1905.

[32] K. Maurin. The Riemann Legacy. Riemann Ideas in Mathematics and Physics. Kluwer Academic Publishers, Dordrecht, 1997.

[33] P. Nastasi, R. Tazzioli. Toward a scientific and personal biography of Tullio Levi-Civita (18731941). Historia Mathematica, 32 (2005) 203-236.

[34] L. Pizzocchero, Geometria differenziale. In: La matematica in Italia dopo l'Unità. Il periodo tra le due guerre mondiali, Edited by S. Di Sieno, A. Guerraggio, P. Nastasi, Marcos y Marcos, Milano, 1998, pp. 321-379.

[35] G. Ruta. Gabrio Piola and Balance Equations. In: The complete works of Gabrio Piola: Volume I. Advanced Structural Materials vol. 38, pp. 752-784. Springer Int. Publ., 2014.

[36] E. Scholz. Hermann Weyl's Contribution to Geometry, 1917-1923. In: The Intersection of History and Mathematics, Edited by S. Chikara, S. Mitsuo, J.W. Dauben, Birkhäuser, Basel, 1994, pp. 203-230.

[37] A. Sommerfeld. Vorlesungen über Theoretische Physik. Band 1: Mechanik, Becker \& Erler, Leipzig, 1943. Italian translation: Lezioni di Fisica Teorica. vol. 1: Meccanica, Sansoni, Firenze, 1957.

[38] R. Tazzioli. Ether and theory of elasticity in Beltrami's work, Archive for history of exact sciences, 46 (1993), 1-37.

[39] S.P. Timoshenko. History of strength of materials, with a brief account of the history of theory of elasticity and theory of structures. McGraw-Hill, New York, 1953.

[40] I. Todhunter, K. Pearson. A history of the theory of elasticity and of the strength of materials, from Galilei to the present time. Cambridge, at the University Press, 1893.

[41] P. Varignon. Nouvelle mécanique ou statique. C. Jombert, Paris, 1725. 